\title{
Dynamics Evolution of Credit Risk Contagion in the CRT Market
}

\author{
Tingqiang Chen, Jianmin He, and Qunyao Yin \\ School of Economics and Management, Southeast University, Nanjing, Jiangsu 211189, China \\ Correspondence should be addressed to Tingqiang Chen; tingqiang88888888@163.com
}

Received 3 October 2012; Revised 4 December 2012; Accepted 20 December 2012

Academic Editor: Qingdu Li

Copyright (C) 2013 Tingqiang Chen et al. This is an open access article distributed under the Creative Commons Attribution License, which permits unrestricted use, distribution, and reproduction in any medium, provided the original work is properly cited.

\begin{abstract}
This work introduces a nonlinear dynamics model of credit risk contagion in the credit risk transfer (CRT) market, which contains time delay, the contagion rate of credit risk, and nonlinear resistance. The model depicts the dynamics behavior characteristics of evolution of credit risk contagion through numerical simulation. Meanwhile, numerical simulations show that, in the CRT market, the contagion rate of credit risk and the nonlinear resistance among CRT activities participants have some significant effects on the dynamics behaviors of evolution of credit risk contagion. Specifically, on the one hand, we find that the status curve of credit risk contagion that causes some significant changes with the increase in the contagion rate of credit risk, moreover, emerges a series of Hopf bifurcation and chaotic phenomena in the process of credit risk contagion. On the other hand, Hopf bifurcation and chaotic phenomena appear in advance with the increase in the nonlinear resistance coefficient and time-delay. In addition, there are a series of periodic windows in the chaotic interval inside, including Hopf bifurcation, inverse bifurcation, and chaos.
\end{abstract}

\section{Introduction}

Over the past few years, with the significant development of nonlinear science, economists have gradually started to use nonlinear theory to study the complex phenomena of social economic system [1-7]. Some far-sighted economists began to apply the nonlinear science research results into economics, which produced the nonlinear economics and the chaos economics. The latest studies of nonlinear theory show that whether interpersonal network, computer network, ecological system, economic system, or disease spread, computer virus spread, forest fire spread, risk spread, complex nonlinear dynamics phenomena, and so forth, can be observed in these social phenomena $[8,9]$. The aforementioned phenomena present complex dynamical behavior, involving Hopf bifurcation, inverse bifurcation, chaos, and fractals. Among these behavior types, chaos and bifurcation are complex phenomena that exist in the nonlinear financial system and are important issues in economic and financial dynamics research [10]. Credit risk transfer (CRT) market is a thirdparty market that connects with the credit markets, the securities market, and the insurance market, in which credit risk contagion has some complex nonlinear characteristics obviously.

At present, participants of the CRT market covered mainly universal banks, commercial banks, securities dealers, insurance companies, investment funds, and parts of nonfinancial institutions. Among them exist close and complicated network relations directly or indirectly, and that constituted a nonlinear giant system. Because the interactions between individuals that have complex nonlinear dynamic properties. Moreover, credit risk contagion is dependent on CRT behaviors of participants of the CRT market and market information dissemination of the relationship network. With the rapid development of the CRT market, the quantity of participants, and the depth and breadth of CRT trading all rapidly increase. This will lead to the increase in the complexity of the CRT market and make the distribution of information and risk of terminal undertaker of credit risk change more complicate. Meanwhile, the rapid development structured products will also increase the complexity. These will make the financial institutions extremely easily cause the superposition or clustering of credit risk in credit risk transfer and cause credit risk contagion. However, credit risk 
contagion also has complex nonlinearity. It will increase the difficulty of the prediction and control of credit risk in CRT market and bring great challenges to credit risk management departments.

Generally, some participants do not fully understand the potential risk of CRT market or lack of corresponding risk management ability into the CRT market, which will lead to some new risks in the process of CRT behaviors. Moreover, the systemic risk can increase in the CRT market. In the imperfect competition market, CRT behaviors not only did not spread risk, but also added to the system risk and increased the likelihood of the credit risk contagion [11]. The existing literature also showed that the rapid development of the CRT market increased the possibility of credit risk contagion across departments and trade. For example, credit risk transfer in creating contagion between banking and insurance systems and caused contagion, and the spread in systemic risk made everybody worse off. At the same time, credit risk transfer induced insurance companies to hold the same assets as banks [12]. Banks' motive of extensive using CDS (Credit Default Swap) is that improve the diversification of their credit risk. However, this might reduce banks' stability. The main reasons behind these negative impacts are firstly, that banks are induced to increase their investment in an illiquid, risky credit portfolio and secondly, that these CDS create a possible channel of credit risk contagion [13].

The theory and practice have recognized the serious consequences of the credit default contagion by the US subprime mortgage crisis in 2008. Moreover, a number of studies are also aware of credit risk contagion in the CRT process [11-14]. At present, the study of credit risk contagion mainly focus on the interbank market and credit market. However, the existing credit risk model have not yet discussed and involved nonlinear dynamic problems of the risk contagion process. However, nonlinear dynamic behaviors are obvious in credit risk contagion due to the complex network relationships, the continuous innovation of CRT tools, and the asymmetric information in CRT market. Moreover, network relations of CRT market exist time delay and nonlinear resistance. Therefore, we try to put the nonlinear system theory into the study of the credit risk contagion in CRT market and construct the nonlinear dynamic model of credit risk contagion in CRT market. Then, we conduct numerical simulation to analyze the dynamic behaviors characteristics of evolution of credit risk contagion in CRT market.

The remainder of this paper is organized as follows. In Section 2, the model of credit risk contagion in CRT market and dynamics behavior characteristics of evolution of credit risk contagion are discussed through numerical simulation. In Section 3, we discuss the bifurcation and chaotic behaviors of credit risk contagion. Finally, we conclude the paper in Section 4.

\section{Dynamics Evolution of Credit Risk Contagion Based on the Vector Field}

With the development of network theory, a number of studies have taken into account the spread and response characters of events in a long-distance connection of network. Newman and Watts [15], Moukarzel [16] have given the dynamic model of constant speed transmission of the events in the network. However, they have not taken into account time-delay and various nonlinear factors. Yang [17] took into account the nonlinear factor and time-delay of events in the long connection and constructed the reasonable dynamic model.

2.1. The Contagion Model of Credit Risk in the CRT Market. We are enlightened by the works [17-19] and propose the dynamic model of credit risk contagion in the CRT market. On the one hand, we assume that the complex network connections among CRT activities participants are NewmanWatts length scale connections and long-distance connections. On the other hand, we take into account the timedelay and nonlinear resistance of long-distances connection between CRT activities participants. In fact, the model is also a nonlinear time-delay differential equation. Therefore, the dynamic model of credit risk contagion is described by the following time-delay differential equation:

$$
\begin{gathered}
\frac{d N(t)}{d t}=\lambda k_{1}-N(t)+\lambda k_{2} N(t-\tau) \\
-\mu \xi\left[\lambda k_{2} N(t-\tau)\right]^{2} \quad t \geq 0, \\
N(t)=c \quad-\tau \leq t \leq 0,
\end{gathered}
$$

where $N(t)$ denotes the number of CRT activities participants that are infected by credit risk in the CRT market, $\xi$ refers to Newman-Watts length scale, $k_{1}$ is the number of instances that the connection distance from the participant infected by credit risk is a Newman-Watts length scale, $k_{2}$ is the number of instances that the connection distance from the participant infected by credit risk is a long-distance connection, $\lambda$ is the effective contagion rate of credit risk in the CRT market, $\mu$ is the nonlinear resistance coefficient of the relationship network comprising CRT market participants, $c \in \mathbb{R}^{+}$is a real parameter, and $\tau$ is the time-delay of credit risk contagion in the long-distance connection. Therefore, the mechanism of the time-delay and the nonlinear resistance of credit risk contagion in Newman-Watts length scale connection and longdistance connection can be described by the time-delay differential equation (1).

According to the general definition, we can derive the balance position and stable point of credit risk contagion when the left side of equation (1) is equal to zero. In fact, this kind of nonlinear dynamics system can be denoted by equation (1), where balance positions may become unstable, periodic solution and the system vibration may emerge, and the phenomenon of Hopf bifurcation and chaos may occur, along with the change in various parameters [20]. Torelli [21], Liu and Spijker [22] have given a numerical Euler method for the solution of delay differential equation as equation (1). We still use the method in this paper. Now, let the stepsize $h$ is such that $h=\tau / m$ and $\theta \in[0,1]$, where $\tau$ is a time-delay, and $m$ is a positive integer. Therefore, according to the one-point 
collocation rule for delay differential equation (1), we can get

$$
\begin{aligned}
N_{n+1}= & N_{n}+\lambda k_{1} h-h\left[(1-\theta) N_{n}+\theta N_{n+1}\right] \\
& +\lambda k_{2} h\left[(1-\theta) N_{n-m}+\theta N_{n-m+1}\right] \\
& -\mu \xi \lambda^{2} k_{2}^{2} h\left[(1-\theta) N_{n-m}+\theta N_{n-m+1}\right]^{2},
\end{aligned}
$$

where $N_{n}$ denotes the approximate value of $N(t)$ at the point $t_{n}$. Let $=(m-\delta) h+h / 2(0 \leq \delta<1)$, then $\Omega_{h}=\left\{t_{n}=n h, n \in\right.$ $Z$ \}. Thus, we can get $t_{n}+\theta h \in\left[t_{n}, t_{n+1}\right]$ and $t_{n}+\theta h-\tau \in$ $\left[t_{n-m}, t_{n-m+1}\right]$. We apply the $\theta$-collocation method to define the approximate value of $N(t)$ at the point $t_{n}+\theta h$ and $t_{n}+$ $\theta h-\tau$ as follows:

$$
\begin{gathered}
N\left(t_{n}+\theta h\right) \approx \theta\left[N\left(t_{n}\right)+N\left(t_{n+1}\right)\right], \\
N\left(t_{n}+\theta h-\tau\right) \approx \theta\left[N\left(t_{n-m}\right)+N\left(t_{n-m+1}\right)\right] .
\end{gathered}
$$

We apply the midpoint collocation method (one-point collocation with $\theta=1 / 2$ ) to equation (1), and can get

$$
\begin{aligned}
N_{n+1}= & N_{n}+\lambda k_{1} h-h\left[\frac{N_{n}+N_{n+1}}{2}\right] \\
& +\lambda k_{2} h\left[\frac{N_{n-m}+N_{n-m+1}}{2}\right] \\
& -\mu \xi \lambda^{2} k_{2}^{2} h\left[\frac{N_{n-m}+N_{n-m+1}}{2}\right]^{2} .
\end{aligned}
$$

Namely,

$$
\begin{aligned}
N\left(t_{n+1}\right)= & N\left(t_{n}\right)+\lambda k_{1} h-h\left[\frac{N\left(t_{n}\right)+N\left(t_{n+1}\right)}{2}\right] \\
& +\lambda k_{2} h\left[\frac{N\left(t_{n-m}\right)+N\left(t_{n-m+1}\right)}{2}\right] \\
& -\mu \xi \lambda^{2} k_{2}^{2} h\left[\frac{N\left(t_{n-m}\right)+N\left(t_{n-m+1}\right)}{2}\right]^{2} .
\end{aligned}
$$

Put equation (3) into equation (5), we can get

$$
\begin{aligned}
N\left(t_{n+1}\right) \approx & N\left(t_{n}\right)+\lambda k_{1} h-h N\left(t_{n}+\frac{h}{2}\right) \\
& +\lambda k_{2} h N\left(t_{n}+\frac{h}{2}-\tau\right) \\
& -\mu \xi \lambda^{2} k_{2}^{2} h\left[N\left(t_{n}+\frac{h}{2}-\tau\right)\right]^{2} .
\end{aligned}
$$

We put $h=\tau / m$ into equation (6), we can get

$$
\begin{aligned}
N\left(t_{n+1}\right) \approx & N\left(t_{n}\right)+\frac{\lambda k_{1} \tau}{m}-\frac{\tau}{m} N\left(t_{n}+\frac{\tau}{2 m}\right) \\
& +\frac{\lambda k_{2} \tau}{m} N\left(t_{n}+\frac{(1-2 m) \tau}{2 m}\right) \\
& -\frac{\mu \xi \lambda^{2} k_{2}^{2} \tau}{m}\left[N\left(t_{n}+\frac{(1-2 m) \tau}{2 m}\right)\right]^{2} .
\end{aligned}
$$

To understand the effect of nonlinear factors on credit risk contagion further, we have to use equation (7) to conduct numerical simulations under the given parameters $\mu, \xi, k_{1}, k_{2}$, $\lambda$, and $\tau$ and the initial condition $N(t)=c(-\tau \leq t \leq 0)$.

2.2. Simulation Analysis of the Dynamics Behavior of Evolution of Credit Risk Contagion in the CRT Market. We try to describe the dynamics behavior characteristics of evolution of credit risk contagion and its influencing factors by the nonlinear time-delayed differential equation in this paper. According to the solving process of equation (1), we know that parameters $\mu, \xi, k_{1}, k_{2}$, and $\lambda$ and the initial condition $N(t)=c(-\tau \leq t \leq 0)$ will affect the stability of the solution of time-delayed differential equations and the trajectory of the process of credit risk contagion. In order to describe the dynamic behaviors and its influencing factors of the process of credit risk contagion in CRT market, we take parameters $\lambda$ and $\mu$ as the bifurcation parameter. Then, we conduct numerical simulations to the dynamics system (1) and analyze the dynamics behavior of credit risk contagion in CRT market. Let $\tau=1, h=0.01, m=100, \delta=0.5$, $\xi=3, k_{1}=10, k_{2}=25$, and the initial condition $N(t)=$ $2(t \in(-\tau, 0))$. Figure 1 depicts the effect of the effective contagion rate $\lambda$ of credit risk on the trajectory curve of credit risk contagion in the CRT market. We find that the status of credit risk contagion changes gradually from "hyperbolic attenuation" (a piece of the hyperbolic) to "logarithm Gauss attenuation," and the influence strength and range of credit risk contagion emerge nonlinear velocity increasing with the increase in the effective contagion rate $\lambda$ of credit risk in CRT market. However, the influence strength and range attenuate rapidly after a period of time and emerge the fat-tail characteristic. This shows that the effect of the default behaviors of CRT activities participants on other participants weakened gradually after a period of time and the default intensity and default state depend on the company oneself and macroeconomic factors. Figure 2 shows that oscillation amplitude and frequency increase gradually with the increase in the effective contagion rate $\lambda$ of credit risk in CRT market. However, the oscillation will weaken after a period of time. Figure 3 shows that the stable state of credit risk contagion will trend to unstable and emerge periodic solution and Hopf bifurcation with the increase in the effective contagion rate $\lambda$ of credit risk in CRT market. Namely, the contagion amplitude and range of credit risk will emerge periodic oscillation with the increase in the effective rate of credit risk contagion in CRT market. Moreover, the limit cycle radius of the attractive domain increases gradually, and the shape of the limit cycle becomes increasingly irregular, such that the bifurcation and chaos phenomena occur with the increase in the effective contagion rate $\lambda$ of credit risk contagion. In Figure 4, we find that the process of credit risk contagion present different "logarithm Gauss attenuation" feature under the influence of the nonlinear resistance of the relationship network comprising CRT activities participants. In Figure 5, we find that the oscillation of the process of credit risk contagion is not affected with the increase in the nonlinear resistance coefficient $\mu$. However, the number of CRT activities 


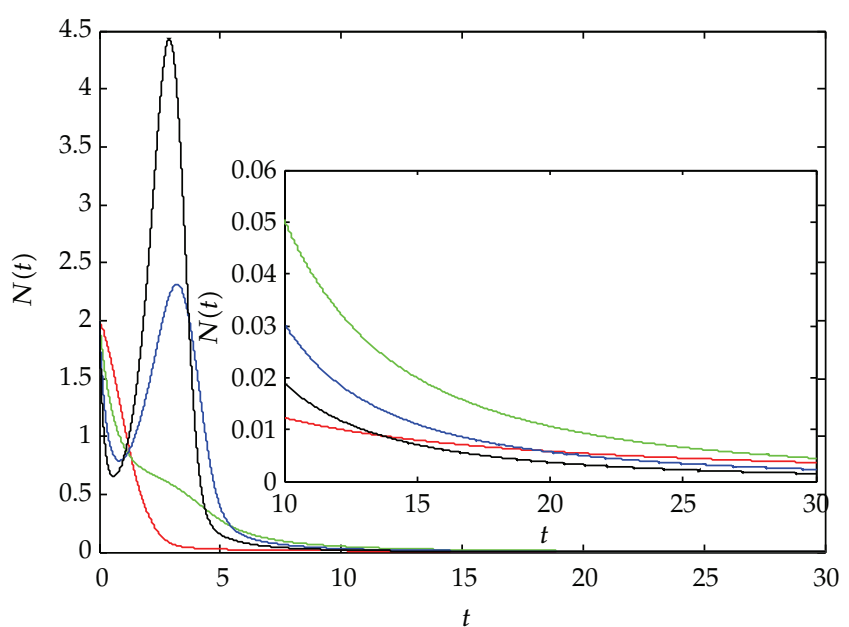

- The contagion rate of credit risk is equal to 0.01

- The contagion rate of credit risk is equal to 0.05

- The contagion rate of credit risk is equal to 0.1

- The contagion rate of credit risk is equal to 0.15

FIGURE 1: The trajectory curve of credit risk contagion where $\mu=$ 0.03 .

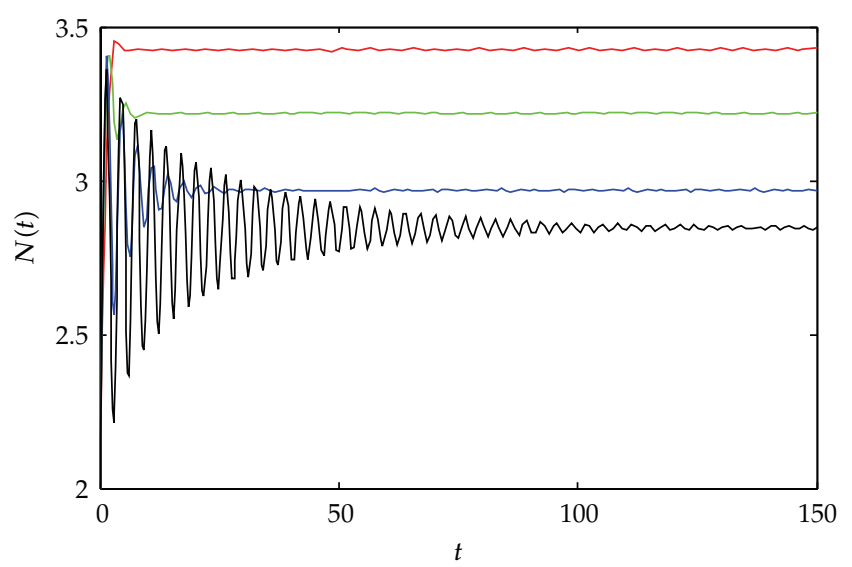

- The contagion rate of credit risk is equal to 0.08

- The contagion rate of credit risk is equal to 0.1

- The contagion rate of credit risk is equal to 0.12

- The contagion rate of credit risk is equal to 0.13

FIGURE 2: The step response of the process of credit risk contagion where $\mu=0.03$.

participants $N(t)$ gradually reduces with the increase in the nonlinear resistance coefficient $\mu$. In Figure 6 , we find that the effect of the nonlinear resistance coefficient $\mu$ on the attract factor of balance position of credit risk contagion, and the number of CRT activities participants $N(t)$ is very sensitive. Namely, the attractive factor of credit risk contagion and the number of CRT activities participants $N(t)$ will decrease rapidly with the increase in nonlinear resistance coefficient $\mu$.

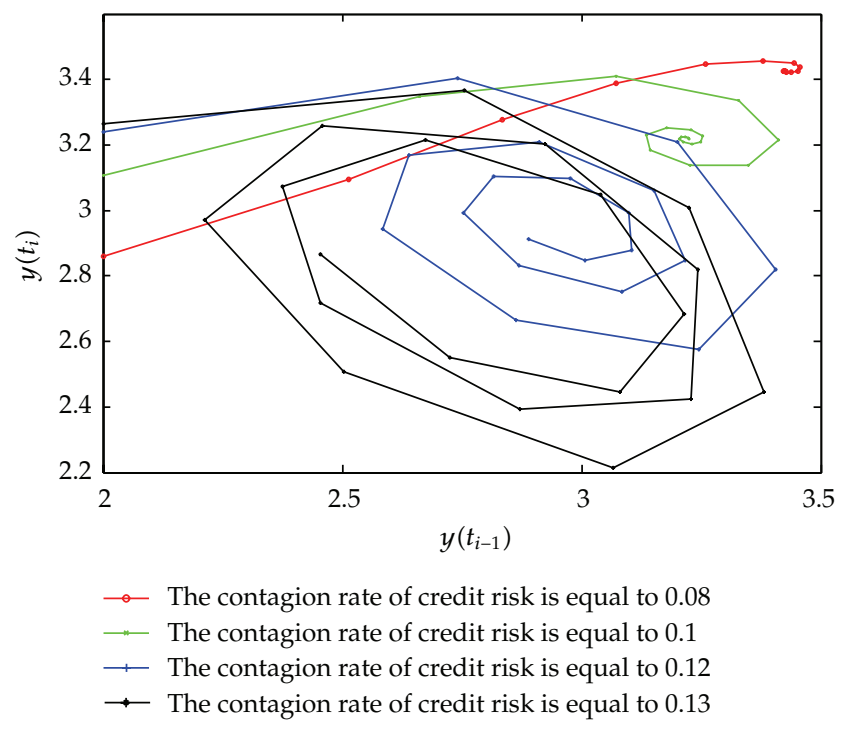

FIGURE 3: The phase diagram of the process of credit risk contagion where $\mu=0.03$.

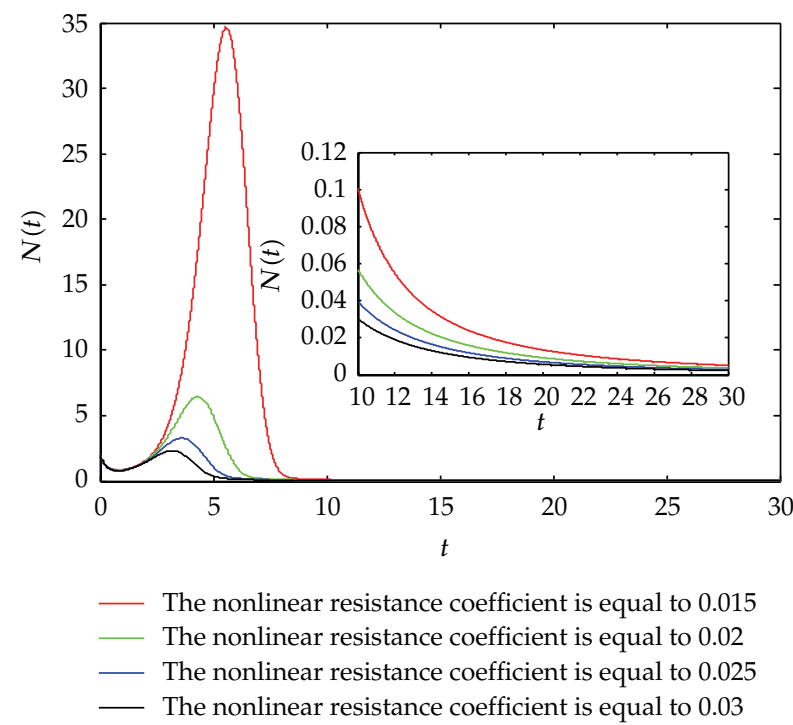

FIGURE 4: The state trajectory curve of the process of credit risk contagion where $\lambda=0.1$.

\section{Bifurcation and Chaotic Analysis of Credit Risk Contagion Based on Logistic Mapping}

3.1. The Model Analysis of Credit Risk Contagion Based on Logistic Mapping. The model (1) of credit risk contagion used the form of vector field to discuss credit risk contagion in credit risk transfer. However, the previous figures are not intuitive and are difficult to interpret. Thus, analyzing the properties of the dynamic system of credit risk contagion, such as the difference of the trajectory curve of period doubling, may be challenging. However, given the intuition, legibility, and geometrical features of the logistic mapping, we often discretize the nonlinear problem of the continuous vector 


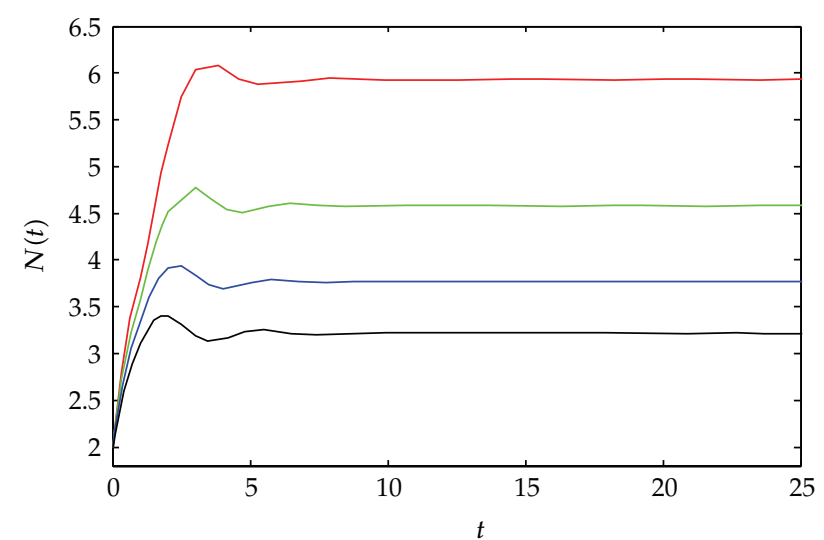

- The nonlinear resistance coefficient is equal to 0.015

- The nonlinear resistance coefficient is equal to 0.02

_ The nonlinear resistance coefficient is equal to 0.025

_ The nonlinear resistance coefficient is equal to 0.03

FIGURE 5: The step response of the process of credit risk contagion where $\lambda=0.1$.

field to the logistic mapping by using a numerical approximation method to analyze the periodic bifurcation and chaos of nonlinear dynamics system. A number of studies use the Euler [23-25] to analyze bifurcation, periodic solution, and chaotic phenomena of nonlinear time-delayed system. We also adopt the Euler method and take step length $h$. Therefore, equation (1) can be transformed into the form following form:

$$
\begin{gathered}
N(t-\tau+h)-N(t-\tau) \\
=h\left\{\lambda k_{1}-N(t)+\lambda k_{2} N(t-\tau)\right. \\
\left.-\mu \xi\left[\lambda k_{2} N(t-\tau)\right]^{2}\right\} .
\end{gathered}
$$

Let $h=\tau, N(t)=N_{n+1}$, and $N(t-\tau)=N_{n}$. Thus, equation (8) can be transformed into the form following form:

$$
N_{n+1}=\frac{\lambda k_{1} \tau}{1+\tau}+\frac{1+\lambda k_{2} \tau}{1+\tau} N_{n}-\frac{\mu \xi \tau \lambda^{2} k_{2}^{2}}{1+\tau}\left(N_{n}\right)^{2}
$$

Therefore, there exists the logistic mapping $f$ as follow:

$$
f: N_{n} \longmapsto N_{n+1}
$$

According to the definition of the fixed point of the logistic mapping, we know that the fixed point of the logistic mapping $f$ should meet $N_{n+1}=N_{n}=N^{*}$. Therefore, we can get the analytic equation of the fixed point of the logistic mapping as follow:

$$
\frac{\mu \xi \tau \lambda^{2} k_{2}^{2}}{1+\tau}\left(N^{*}\right)^{2}-\frac{\lambda k_{2} \tau-\tau}{1+\tau} N^{*}-\frac{\lambda k_{1} \tau}{1+\tau}=0 .
$$

Therefore, we can get the fixed point of the logistic mapping $f$ by equation (11) as follow:

$$
\begin{aligned}
& N_{1}^{*}=\frac{\left(\lambda k_{2} \tau-\tau\right)+\sqrt{\left(\lambda k_{2} \tau-\tau\right)^{2}+4 \mu \xi k_{1} \tau^{2} \lambda^{3} k_{2}^{2}}}{2 \mu \xi \tau \lambda^{2} k_{2}^{2}}, \\
& N_{2}^{*}=\frac{\left(\lambda k_{2} \tau-\tau\right)-\sqrt{\left(\lambda k_{2} \tau-\tau\right)^{2}+4 \mu \xi k_{1} \tau^{2} \lambda^{3} k_{2}^{2}}}{2 \mu \xi \tau \lambda^{2} k_{2}^{2}} .
\end{aligned}
$$

Obviously, $N_{2}^{*}<0$ is unrealistic. Therefore, the fixed point $N_{1}^{*}$ is sole fixed point of the logistic mapping $f$. According to the definition of the logistic mapping and the Lyapunov movement stability, we know that the movement stability of the fixed point depends on the characteristic root of the derived operator of the logistic mapping, which is Floquet multiplier [26, 27]. Therefore, the Floquet multiplier will determine the stability of the fixed point $N_{1}^{*}$. Namely,

$$
\left.D g\right|_{N^{*}}=1-\frac{\sqrt{\left(\lambda k_{2} \tau-\tau\right)^{2}+4 \mu \xi k_{1} \tau^{2} \lambda^{3} k_{2}^{2}}}{1+\tau} .
$$

According to the nonlinear system theory $[27,28]$, if $|D g|_{N^{*}}>1$, then the fixed point $N^{*}$ will become unstable; if $|D g|_{N^{*}}<1$, then the fixed point $N^{*}$ is asymptotically stable; if $|D g|_{N^{*}}=1$, then the fixed point $N^{*}$ is criticality stable. So, for the fixed point $N^{*}$ of the mapping $f$, the fixed point $N^{*}$ is asymptotically stable when $\mu<\left(4(1+\tau)^{2}-\left(\lambda k_{1} \tau-\right.\right.$ $\left.\tau)^{2}\right) / 4 \xi k_{1} k_{2}^{2} \tau^{2} \lambda^{3}$, is criticality stability when $\mu=\left(4(1+\tau)^{2}-\right.$ $\left.\left(\lambda k_{1} \tau-\tau\right)^{2}\right) / 4 \xi k_{1} k_{2}^{2} \tau^{2} \lambda^{3}$, or is unstable when $\mu>\left(4(1+\tau)^{2}-\right.$ $\left.\left(\lambda k_{1} \tau-\tau\right)^{2}\right) / 4 \xi k_{1} k_{2}^{2} \tau^{2} \lambda^{3}$.

According to the nonlinear dynamic related theory [2628], if there exists a series of period-doubling bifurcation phenomena, then a series of period-doubling bifurcation leads to chaos. In recent years, much works used topological horseshoes embedded method to study chaos rigorously [28$34]$. By this method, one can not only prove the existence of chaos, but also reveal the mechanism of chaotic phenomena by showing the structure of chaotic attractors [31-34]. Beyond that, some works used the Lyapunov exponents [35] and set oriented numerical methods $[36,37]$ to prove the existence of chaos. Li and Yorke [38] gave a definition of chaos that the existence of a point of period 3 implies the existence of chaos. Therefore, according to this definition, we use numerical simulation to discuss the fixed point and its stability, bifurcation, and chaos of the mapping from the intuitive.

3.2. Numerical Simulation Analysis. Let $\xi=3, \tau=1, k_{1}=10$, $k_{2}=25$, and the initial condition $N(t)=2(t \in(-\tau, 0))$. We use equation (8) to conduct numerical simulations. The Figure 3 reflects the Hopf bifurcation process and its variation characteristics of credit risk contagion with parameter $\lambda$ and $\mu$. Figures 7(a) and 7(b) reflect the Hopf bifurcation and chaos characteristics of credit risk contagion with the increase in the effective contagion rate $\lambda$ of credit risk. Figure 7 (a) reflects that the process of credit risk contagion exists the only stable constant state when parameter $\lambda$ is kept at a proper level. 


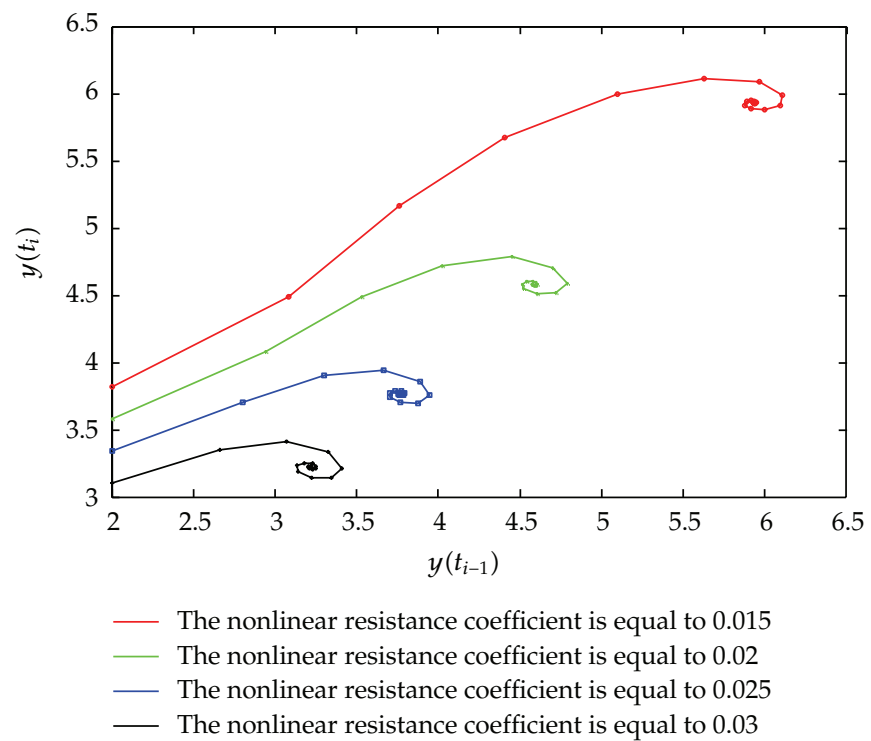

FIGURE 6: The phase diagram of the process of credit risk contagion where $\lambda=0.1$.

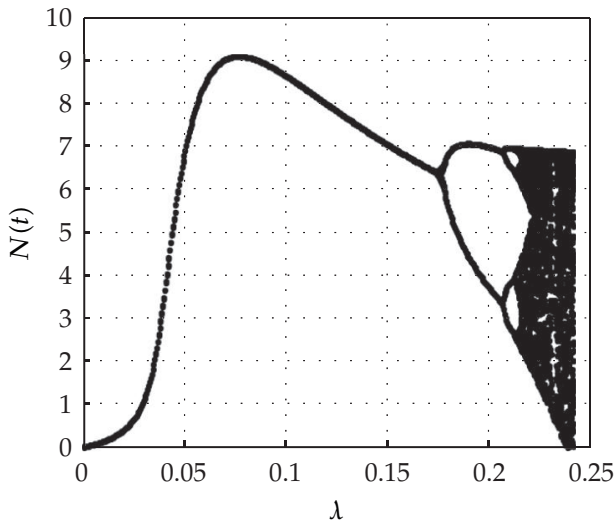

(a)

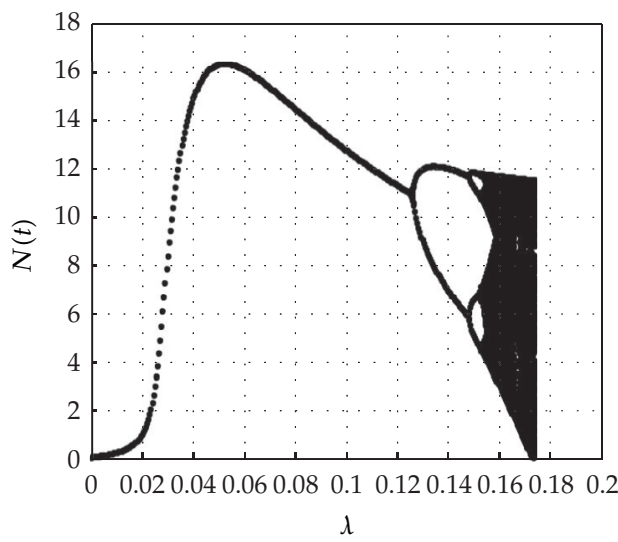

(c)

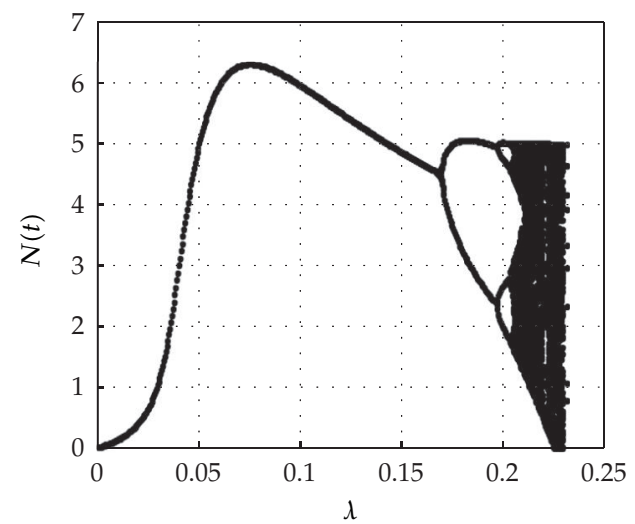

(b)

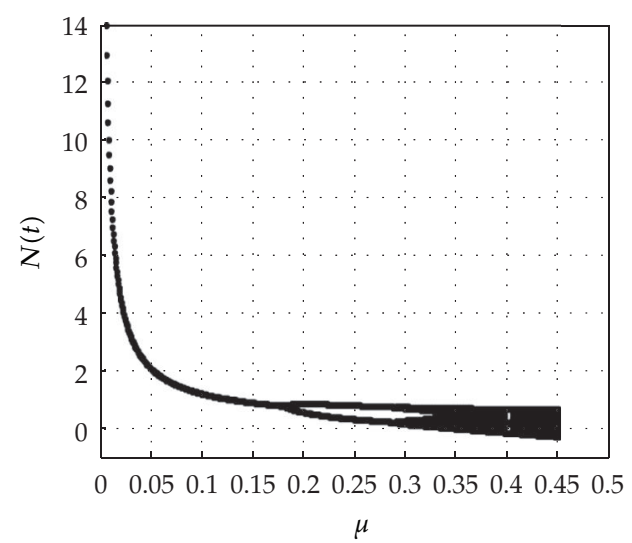

(d)

FIgURE 7: (a) The bifurcation diagram of the process of credit risk contagion with $\lambda$ when $\mu=0.01$; (b) the bifurcation diagram of the process of credit risk contagion with $\lambda$ when $\mu=0.015$; (c) the bifurcation diagram of the process of credit risk contagion with $\lambda$ when $\mu=0.01$, $\tau=1.5$; (d) the bifurcation diagram of the process of credit risk contagion with $\mu$ when $\lambda=0.1$. 


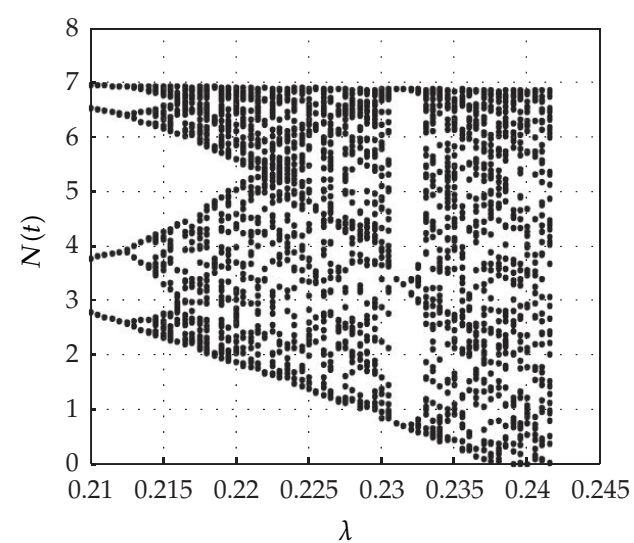

(a)

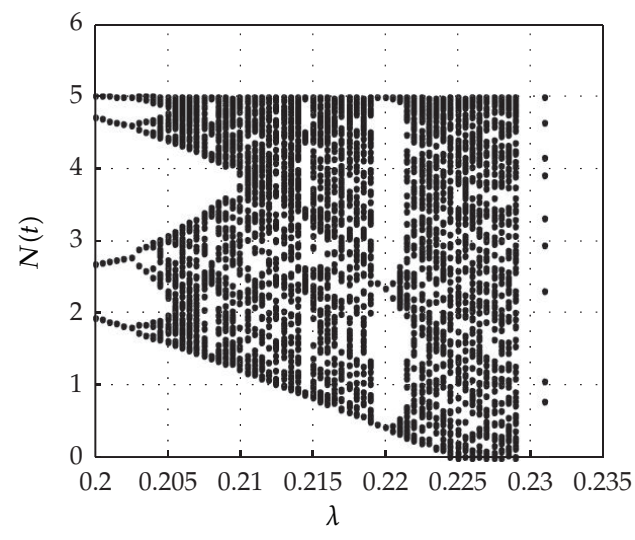

(b)

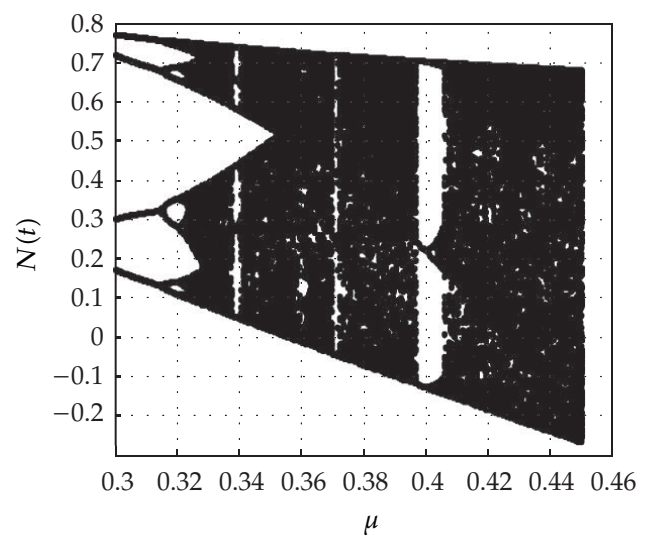

(c)

FIGURE 8: (a) The bifurcation diagram in the chaos area when $\mu=0.01$; (b) the bifurcation diagram in the chaos area when $\mu=0.015$; (c) the bifurcation diagram in the chaos area when $\lambda=0.1$.

Moreover, the process of credit risk contagion emerge different types of period bifurcation and periodic oscillation with the increase in the effective contagion rate $\lambda$ of credit risk in CRT market. According to the definition of Li-Yorke [29], the process of credit risk contagion can occur chaos phenomenon when the effective contagion rate $\lambda$ reaches to a proper value. Figure 7(b) reflects a series of similar characteristics with Figure 7(a). However, we also find that the Hopf bifurcation and chaotic phenomena of credit risk contagion emerge in advance with the increase in the nonlinear resistance coefficient $\mu$. In Figure 7 (c), we find that the Hopf bifurcation and chaotic phenomena of credit risk contagion emerge in advance with the increase in time-delay $\tau$. In Figure $7(\mathrm{~d})$, we find that the process of credit risk contagion exists the only stable constant state when parameter $\lambda$ is kept at a proper level. Moreover, the process of credit risk contagion emerges different types of period bifurcation and periodic oscillation with the increase in the nonlinear resistance coefficient $\mu$ among CRT activities participants. According to the definition of Li-Yorke [29], the process of credit risk contagion can occur chaos phenomenon when the nonlinear resistance coefficient $\mu$ reaches to a proper value.

According to numerical simulation and comparative analysis, we find that the process of credit risk contagion can emerge three states, including the stable constant state, Hopf bifurcation, and chaos with the increase in parameter $\lambda$ and $\mu$. However, these cannot more directly depict the nonlinear dynamic behavior characteristics after occurring chaotic phenomena. Therefore, we further discuss the effect of these parameters on the chaotic state and the period window of the process of credit risk contagion. In Figures 8(a) and 8(b), we find that Hopf bifurcation, pour bifurcation, and chaos mixed emerge in chaotic interval internal period window. Moreover, Hopf bifurcation, pour bifurcation, and chaos phenomena emerge in advance in chaos interval inside with the increase in nonlinear resistance coefficient $\mu$. Figure 8(c) shows that chaos states are significant in the process of credit risk contagion with the increase in nonlinear resistance coefficient $\mu$. However, Hopf bifurcation and pour bifurcation features become relatively obscure comparing to the Figures 8(a) and $8(\mathrm{~b})$.

\section{Conclusion}

In this paper, we constructed a nonlinear dynamic model of credit risk contagion based on literatures [17-19]. Moreover, the dynamical properties of the nonlinear dynamics system 
of credit risk contagion were investigated. We found that the effective rate of credit risk contagion and nonlinear resistance between CRT market participants have significant effect on dynamics behavior of credit risk contagion. Moreover, we found a series of complex Hopf bifurcation, inverse bifurcation, and chaos phenomena in the nonlinear dynamics system of credit risk contagion through a numerical simulation. At the same time, there are a series of period window in chaos interval inside, and that emerge intertwined state including Hopf bifurcation, pour bifurcation, and chaos. The study of dynamics behavior of evolution of credit risk contagion can help us to understand the effect of the interaction between the internal nonlinear factors and external disturbance of credit risk contagion, which has important theoretical and practical value.

There is still much work that is worth further research. For example, in the real world, a variety of noises usually influence the process of credit risk contagion and its dynamics behaviors, such as Gaussian noise, random noises, and so forth. For the kind of credit risk contagion with both timedelay and noises, we leave it for the future work.

\section{Acknowledgments}

The authors wish to express their gratitude to the referees for their invaluable comments. This work was supported by the National Natural Science Foundation of China Grant (nos. 71071034, 71173103, and 71201023), the Humanities and Social Science Youth Foundation of the Ministry of Education of China (no. 12YJC630101), the Funding of Jiangsu Innovation Program for Graduate Education (no. CXZZ12-0131), and the Scientific Research Foundation of Graduate School of Southeast University (no. YBJJ1238).

\section{References}

[1] O. Gomes, "Routes to chaos in macroeconomic theory," Journal of Economic Studies, vol. 33, no. 6, pp. 437-468, 2006.

[2] P. Holmes, "A nonlinear oscillator with a strange attractor," Philosophical Transactions of the Royal Society of London, vol. 292, no. 1394, pp. 419-448, 1979.

[3] S. Serrano, R. Barrio, A. Dena, and M. Rodríguez, "Crisis curves in nonlinear business cycles," Communications in Nonlinear Science and Numerical Simulation, vol. 17, no. 2, pp. 788-794, 2012.

[4] B. H. Kim, H. G. Min, and Y. K. Moh, "Nonlinear dynamics in exchange rate deviations from the monetary fundamentals: an empirical study," Economic Modelling, vol. 27, no. 5, pp. 1167$1177,2010$.

[5] T. O. Awokuse and D. K. Christopoulos, "Nonlinear dynamics and the exports-output growth nexus," Economic Modelling, vol. 26, no. 1, pp. 184-190, 2009.

[6] W. Wu, Z. Chen, and W. H. Ip, "Complex nonlinear dynamics and controlling chaos in a Cournot duopoly economic model," Nonlinear Analysis: Real World Applications, vol. 11, no. 5, pp. 4363-4377, 2010.

[7] L. F. Petrov, "Nonlinear effects in economic dynamic models," Nonlinear Analysis: Theory Methods \& Applications, vol. 71, no. 12, pp. e2366-e2371, 2009.
[8] B. Liu, Y. Duan, and S. Luan, "Dynamics of an SI epidemic model with external effects in a polluted environment," Nonlinear Analysis: Real World Applications, vol. 13, no. 1, pp. 27-38, 2012.

[9] S. Reitz and M. P. Taylor, "The coordination channel of foreign exchange intervention: a nonlinear microstructural analysis," European Economic Review, vol. 52, no. 1, pp. 55-76, 2008.

[10] B. Xin, J. Ma, and Q. Gao, "The complexity of an investment competition dynamical model with imperfect information in a security market," Chaos, Solitons \& Fractals, vol. 42, no. 4, pp. 2425-2438, 2009.

[11] F. Allen and D. Gale, "Systemic risk and regulation," Wharton Financial Institutions Center Working Paper No. 95-124, 2005.

[12] F. Allen and E. Carletti, "Credit risk transfer and contagion," Journal of Monetary Economics, vol. 53, no. 1, pp. 89-111, 2006.

[13] U. Neyer and F. Heyde, "Credit default swaps and the stability of the banking sector," International Review of Finance, vol. 10, no. 1, pp. 27-61, 2010.

[14] T. Santos, "Comment on: credit risk transfer and contagion," Journal of Monetary Economics, vol. 53, no. 1, pp. 113-121, 2006.

[15] M. E. J. Newman and D. J. Watts, "Scaling and percolation in the small-world network model," Physical Review E, vol. 60, no. 6, pp. 7332-7342, 1999.

[16] C. F. Moukarzel, "Spreading and shortest paths in systems with sparse long-range connections," Physical Review E, vol. 60, no. 6, part A, pp. R6263-R6266, 1999.

[17] X. S. Yang, "Chaos in small-world networks," Physical Review E, vol. 63, no. 4, Article ID 046206, 4 pages, 2001.

[18] S. R. Pastor, A. Vázquez, and A. Vespignani, "Dynamical and correlation properties of the internet," Physical Review Letters, vol. 87, no. 25, Article ID 258701, 4 pages, 2001.

[19] S. R. Pastor and A. Vespignani, "Epidemic dynamics and endemic states in complex networks," Physical Review E, vol. 63, no. 6, Article ID 066117, 8 pages, 2001.

[20] J. Guckenheimer and P. Holmes, Nonlinear Oscillations, Dynamical Systems, and Bifurcations of Vector Fields, vol. 42 of Applied Mathematical Sciences, Springer, Berlin, Germany, 1990.

[21] L. Torelli, "Stability of numerical methods for delay differential equations," Journal of Computational and Applied Mathematics, vol. 25, no. 1, pp. 15-26, 1989.

[22] M. Z. Liu and M. N. Spijker, "The stability of the $\theta$-methods in the numerical solution of delay differential equations," IMA Journal of Numerical Analysis, vol. 10, no. 1, pp. 31-48, 1990.

[23] N. J. Ford and V. Wulf, "The use of boundary locus plots in the identification of bifurcation points in numerical approximation of delay differential equations," Journal of Computational and Applied Mathematics, vol. 111, no. 1-2, pp. 153-162, 1999.

[24] T. Koto, "Periodic orbits in the Euler method for a class of delay differential equations," Computers \& Mathematics with Applications, vol. 42, no. 12, pp. 1597-1608, 2001.

[25] M. Peng, "Bifurcation and chaotic behavior in the Euler method for a Uçar prototype delay model," Chaos Solitons Fractals, vol. 22, no. 2, pp. 483-493, 2004.

[26] R. Seydel, Practical Bifurcation and Stability Analysis: From Equilibrium to Chaos, Springer, 1999.

[27] D. Rugh, Nonlinear System Theory, The John Hopkins University Press, Baltimore, Md, USA, 1980.

[28] S. Wiggins, Introduction to Applied Nonlinear Dynamical Systems and Chaos, vol. 2 of Texts in Applied Mathematics, Springer Academic Press, New York, NY, USA, 1990. 
[29] J. Kennedy and J. A. Yorke, "Topological horseshoes," Transactions of the American Mathematical Society, vol. 353, no. 6, pp. 2513-2530, 2001.

[30] X.-S. Yang and Y. Tang, "Horseshoes in piecewise continuous maps," Chaos, Solitons \& Fractals, vol. 19, no. 4, pp. 841-845, 2004.

[31] Q. Li, "A topological horseshoe in the hyperchaotic Rössler attractor," Physics Letters A, vol. 372, no. 17, pp. 2989-2994, 2008.

[32] Q. Li and X.-S. Yang, "A simple method for finding topological horseshoes," International Journal of Bifurcation and Chaos in Applied Sciences and Engineering, vol. 20, no. 2, pp. 467-478, 2010.

[33] Q. Li and X.-S. Yang, "New walking dynamics in the simplest passive bipedal walking model," Applied Mathematical Modelling, vol. 36, no. 11, pp. 5262-5271, 2012.

[34] Q. Li and X. Yang, "Two kinds of horseshoes in a hyperchaotic neural network," International Journal of Bifurcation and Chaos, vol. 22, no. 8, Article ID 1250200, 14 pages, 2012.

[35] K. Tomasz, M. Konstantin, and M. Marian, Computational homology, vol. 157 of Applied Mathematical Sciences, Springer, New York, NY, USA, 2004.

[36] T. Csendes, B. M. Garay, and B. Bánhelyi, "A verified optimization technique to locate chaotic regions of Hénon systems," Journal of Global Optimization, vol. 35, no. 1, pp. 145-160, 2006.

[37] S. Sertl and M. Dellnitz, "Global optimization using a dynamical systems approach," Journal of Global Optimization, vol. 34, no. 4, pp. 569-587, 2006.

[38] T. Y. Li and J. A. Yorke, "Period three implies chaos," The American Mathematical Monthly, vol. 82, no. 10, pp. 985-992, 1975. 




Advances in

Operations Research

mansans

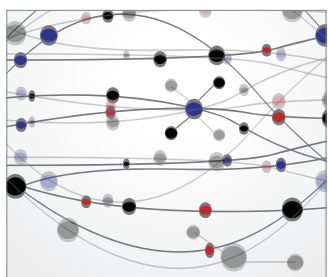

The Scientific World Journal
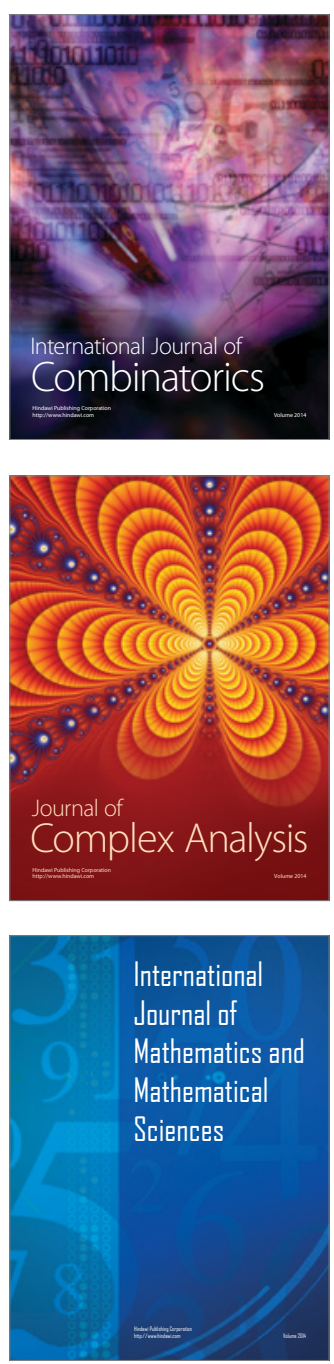
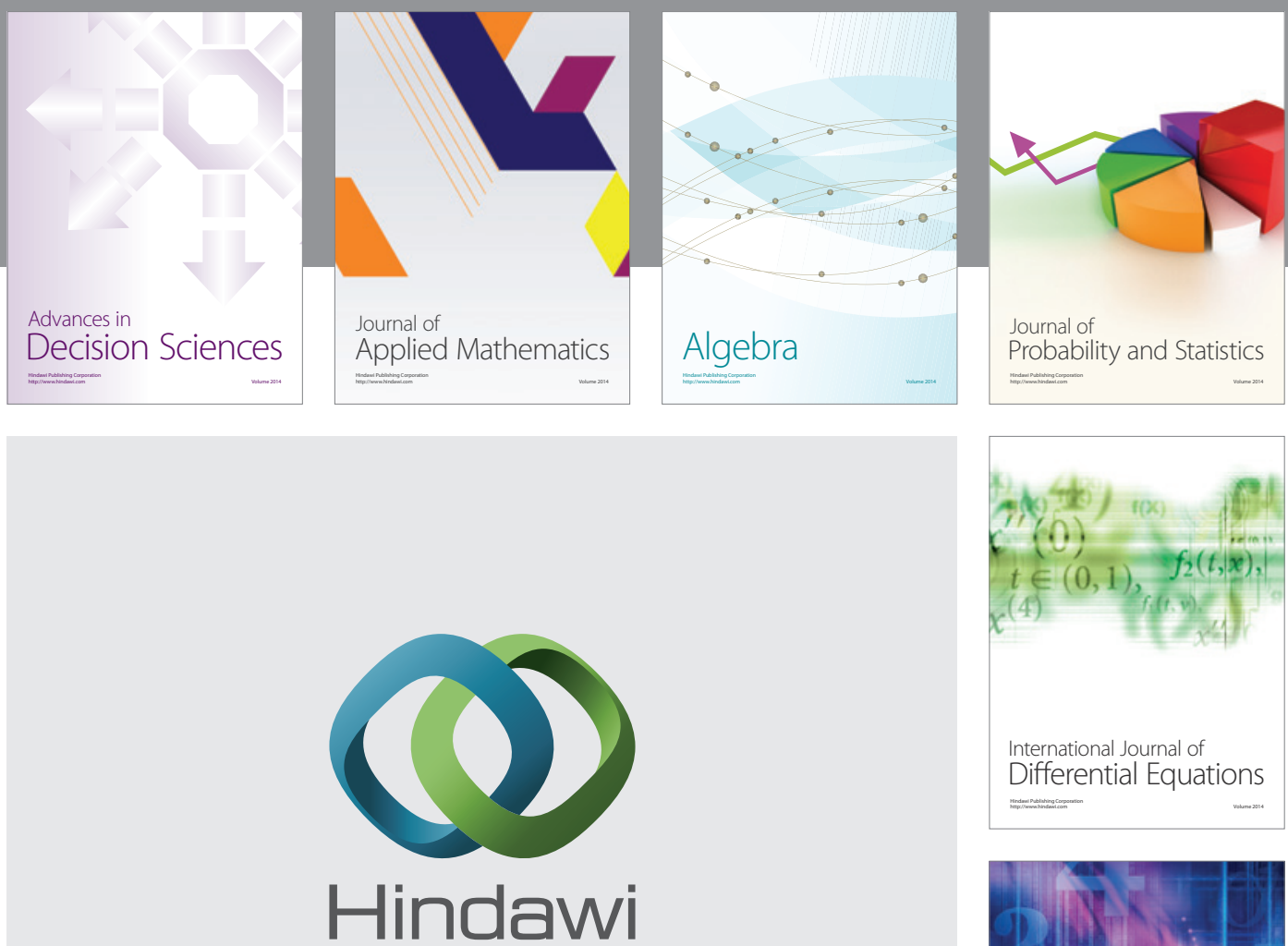

Submit your manuscripts at http://www.hindawi.com
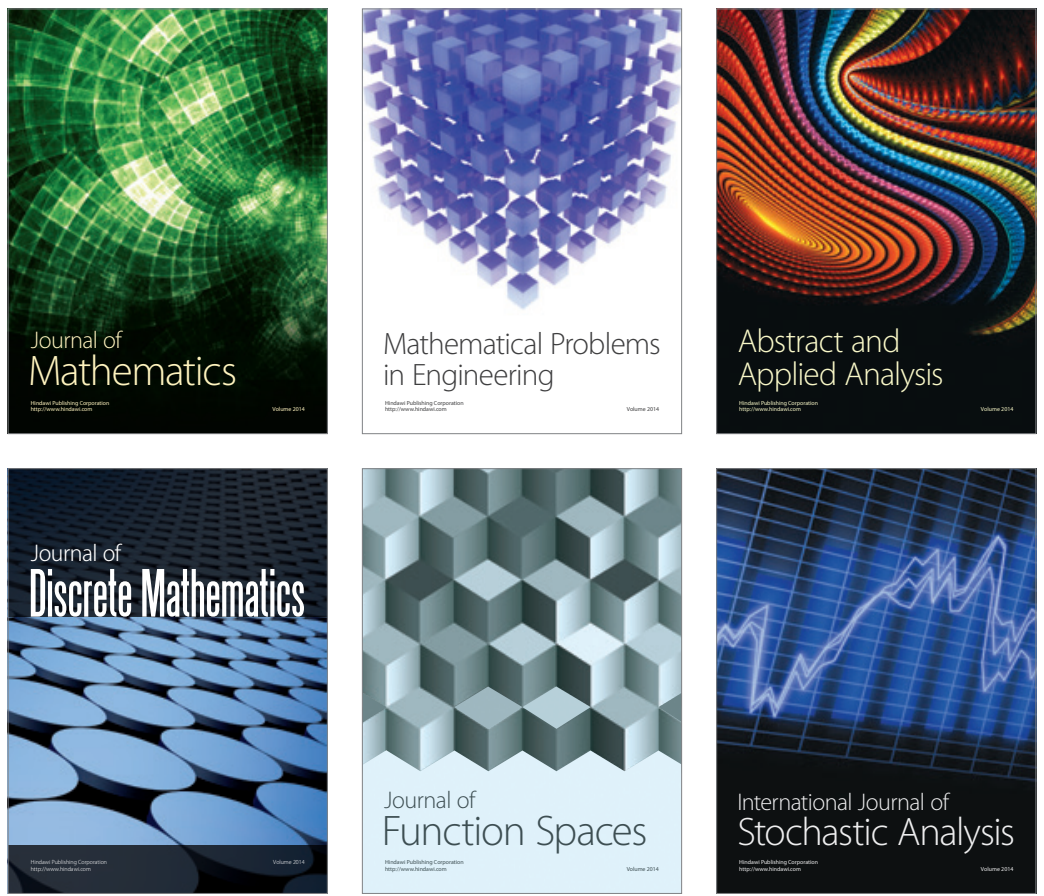

Journal of

Function Spaces

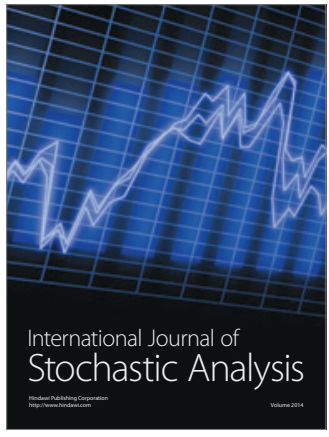

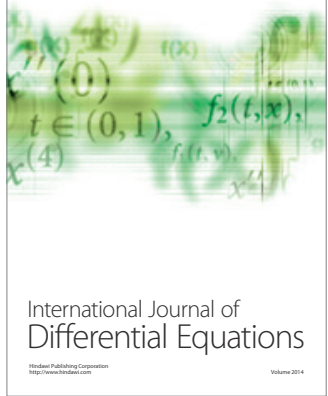
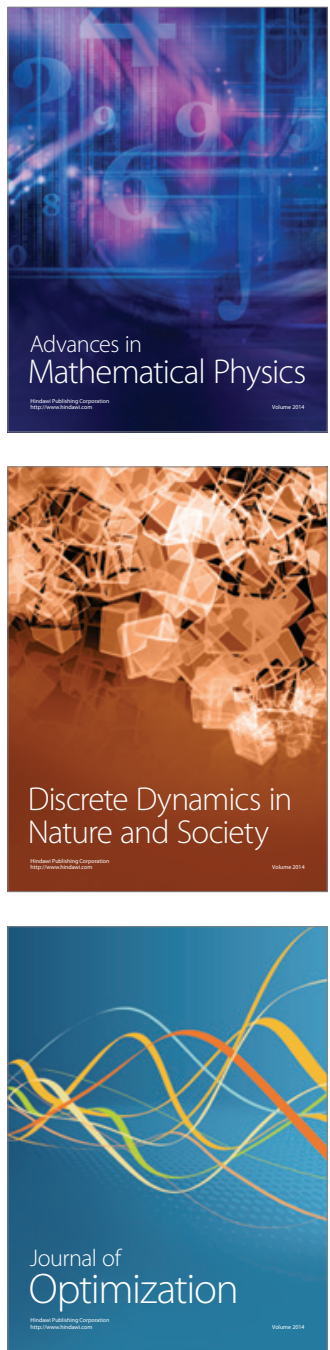